\title{
DEVELOPMENT AND VALIDATION OF E-BUS SIMULATION MODEL
}

\author{
Marko Stokić ${ }^{1}$, Marko Đogatović ${ }^{2}$ \\ 1,2 University of Belgrade, Faculty of Transport and Traffic Engineering, Vojvode Stepe 305, \\ 11000 Belgrade, Serbia
}

Received 29 November 2020; accepted 20 January 2021

\begin{abstract}
In this paper, a simulation model of an electric bus Higer KLQ6125GEV3 is developed in IGNITE software taking into account technical-operational characteristics and drive cycle which is achieved during real operating conditions. The goal of this paper is a validation of the considered model based on the comparison of the generated power from the electric motor, which was recorded in real operating conditions, with the generated power from the electric motor obtained in the simulation program. Through multiple simulations with different parameters, an almost identical drive cycle was obtained in the simulation compared to the recorded drive cycle in real operating conditions, which further resulted in very high degree of correlation between recorded power and power obtained in the simulation. In this way, the simulation model of Higer KLQ6125GEV3 bus was validated and can be used for examination of the technical-operational characteristics of the considered bus on other lines of public transport in order to determine the convenience of its application on them.
\end{abstract}

Keywords: simulation, validation, electric bus, IGNITE.

\section{Introduction}

Countries and their cities around the world are increasingly struggling with air pollution caused by transport sector (Walmsley et al., 2015; Kumar et al., 2018; Huang et al., 2019). Transport sector has the major impact on energy consumption and air pollution (Torok, 2015; Solaymani, 2019), where the road transport has share in total transport in the EU over 75\% (Eurostat, 2019). One of the important polluters in road transport sector are vehicles of public passenger transport (Xylia and Silveira, 2017). The potential strategy to reduce air pollution in this sector is introducing alternative fuel vehicles (Xylia and Silveira, 2017).
Certainly, one of the most considered type of alternative propulsion energy is electricity (Velazquez et al., 2015; Emberger, 2017; Fu et al., 2019). In this sense, there is a practice throughout Europe of introducing electric buses (Mišanović et al., 2015). According to the European ZeEUS (Zero Emission Urban Bus System) project, at the end of 2017, about 650 electric buses were in operation in 88 cities in Europe (including buses with so-called "plug-in" hybrid drive) (UITP, 2016). Many cities have procured various electric buses in order to test them and test their suitability on various public transport lines. Since this type of testing requires a significant financial investment, as well as significant time that needs to be set aside

${ }^{1}$ Corresponding author: m.stokic@sf.bg.ac.rs 
for testing, and the results do not have to be positive, it is necessary to find a solution that will give sufficiently accurate results and will require significantly less financial and time investments. In that sense, one of the possible solutions is the use of simulation software that will credibly describe the behavior of vehicles in real operating conditions. Such simulation software has multiple benefits such as: conducting multiple experiments, cheaper and safer experiments than in real-world operating conditions, simplicity in comparison to analytical techniques, lack of restrictive assumptions which are usually required by analytical models, and determination of any performance. Moreover, in certain situations using simulations is the only method that can be used to obtain solutions.

For this purpose, the simulation software IGNITE, by Ricardo, was used in this paper. The authors have modeled an electric bus Higer KLQ6125GEV3 in mentioned simulation software. The main contribution of this paper is the validation of a modeled bus in simulation software, in terms of speed profile and generated electric motor (EM) power as a function of time and distance traveled. This would enable testing the suitability of application of this bus through a simulation program on other lines of public transport both in Belgrade and in other cities in the Republic of Serbia.

The paper is structured as follows. The second chapter gives detailed characteristics of the bus itself and the characteristics of bus line on which it operates. The third chapter presents the simulation model of the bus and explains the brief procedure of its modeling, while the fourth chapter presents and compares the obtained results of the speed profile and generated power of simulated electric motors with real operating electric motors. The last chapter gives concluding remarks and directions for future research.

\section{Defining Bus and Line Characteristics}

In order to successfully realize simulation, it is necessary to define the key operational and technical characteristics of the bus Higer KLQ6125GEV3, as well as the characteristics of the bus line on which this vehicle operates.

\subsection{Defining Technical and Operational Characteristics of e-bus}

Under the technical characteristics of the observed bus are defined vehicle dimensions (length, width and height), vehicle weight (own weight and current weight of the vehicle with passengers), its center of gravity (CoG), engine characteristics (power and torque), power transmission system, characteristics of tires (tire diameter, rolling resistance coefficient, pressure), supercapacitor capacity, characteristics of electric motors, other consumers of electricity in the vehicle (e.g. air conditioning, heating system, etc.). All the necessary technical characteristics of the bus needed to make a simulation model were obtained by reviewing the following literature (Mišanović et al., 2018; RDW, 2014). The most important technical characteristics are given in Table 1. On the other hand, the current vehicle speed as a function of time can be pointed out as an important operational characteristic. Based on this parameter it is possible to determine the current vehicle's acceleration and deceleration, engine speed, as well as engine load. All operating characteristics can be determined by recording these parameters in real time in real operating conditions with the help of adequate equipment. 
Table 1

Technical Characteristics of the Higer KLQ6125GEV3 e-bus

\begin{tabular}{|c|c|c|}
\hline \multirow{6}{*}{ Vehicle } & Manufacturer \& type & Higer KLQ6125GEV3 \\
\hline & Dimensions (length/width/height) & $12000 / 2550 / 3630 \mathrm{~mm}$ \\
\hline & Curb weight & $12550 \mathrm{~kg}$ \\
\hline & Vehicle capacity (passengers + driver) & $82+1$ \\
\hline & Gross vehicle weight & $18000 \mathrm{~kg}$ \\
\hline & Maximal speed & $70 \mathrm{~km} / \mathrm{h}$ \\
\hline \multirow{4}{*}{ Axles } & Number of axles & 2 \\
\hline & Wheelbase length & $5900 \mathrm{~mm}$ \\
\hline & Front axle mass (unladen / laden) & $3800 \mathrm{~kg} / 6610 \mathrm{~kg}$ \\
\hline & Rear axle mass (unladen / laden) & $8750 \mathrm{~kg} / 11390 \mathrm{~kg}$ \\
\hline \multirow{4}{*}{ Tires } & Rolling radii & $466 \mathrm{~mm}$ \\
\hline & Wheel rim size & $8.25 \times 22.5$ \\
\hline & Offset center distance & $168 \mathrm{~mm}$ \\
\hline & Tyre pressure & $900 \mathrm{kpa}$ \\
\hline \multirow{2}{*}{ Supercapacitor } & Manufacturer \& type & Aowei U-CAP (37DT6-03210) \\
\hline & Capacity & $20 \mathrm{kWh}$ \\
\hline \multirow{4}{*}{ Power plant } & Number of electric motors & 2 \\
\hline & Manufacturer and type & Siemens Electrical Drives Ltd 1PV5135 \\
\hline & Electric motors power & $\begin{array}{l}2 \times 90 \mathrm{~kW} \text { (peak opt.) } \\
2 \times 60 \mathrm{~kW} \text { (nom. opt.) } \\
\end{array}$ \\
\hline & Electric motors torque & $2 \times 430 \mathrm{Nm}$ \\
\hline \multirow{3}{*}{ Transmission } & Type & Mechanical \\
\hline & Number of gears & 1 \\
\hline & Total gear ratios & 25.79 \\
\hline \multirow{2}{*}{ Inverter } & Manufacturer \& type & Zhonglian IEVD 130-60ZO6GA \\
\hline & Operating voltage & $580 \mathrm{~V} \mathrm{DC} / \mathrm{AC} 500-650 \mathrm{~V}$ \\
\hline \multirow{2}{*}{ Converter } & Manufacturer \& type & Zhonglian DY074C \\
\hline & Operating voltage & 12-24-48 V DC \\
\hline \multirow{5}{*}{$\begin{array}{l}\text { Auxiliary } \\
\text { systems }\end{array}$} & \begin{tabular}{|l|} 
Air-condition \\
\end{tabular} & Thermo king 81DT6 \\
\hline & Pump control & KVD HDZHB 1416 \\
\hline & Compressor & IEM ER 230 \\
\hline & UC-cooler & Aowei 37DT6 \\
\hline & Traction control & Siemens 10DT6 \\
\hline
\end{tabular}

\subsection{Defining Characteristics of Bus Line}

The most important characteristics of the observed bus line, that are necessary for the simulation, are length and slope (longitudinal slope). These parameters were recorded in real operating conditions using a device Garmin GPSMAP 62s. In particular, mentioned device records the current altitude and GPS coordinates with a frequency of $1 \mathrm{~Hz}$ on the basis of which it is possible to determine the longitudinal slope of the road. The final road slope was determined using a combination of IGNITE software and the moving average method, where the mentioned software is used to interpolate recorded altitude points as a function of the traveled distance, after which the moving average method was applied to the obtained values. The appearance of the slope based on recorded data and slope after the application of the software and the moving average method is shown in Figure 1. 


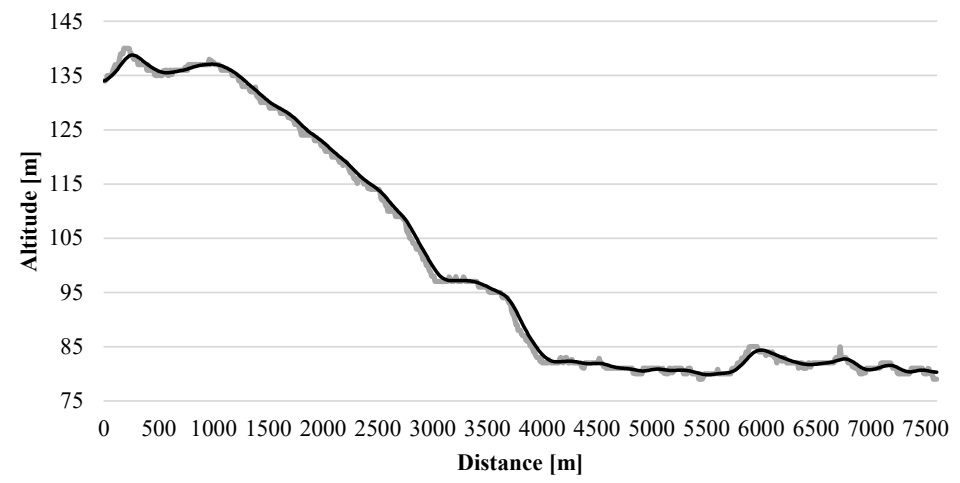

Recorded data

Fig. 1.

The Appearance of the Slope Based on Recorded Data (Grey) and Slope after the Application of the Software and the Moving Average Method (Black)

After the necessary technical and operational characteristics of the Higer KLQ6125GEV3 bus have been defined, as well as the characteristics of the line which the mentioned bus is in operation on, it is possible to approach the simulation modeling of the e-bus. In the next chapter, a brief explanation will be given on how the e-bus was simulated in the IGNITE simulation software.

\section{E-bus Simulation Model}

In this chapter, the layout of the entire Higer KLQ6125GEV3 bus simulation model modeled in the IGNITE software is given (Figure 2). Furthermore, a detailed explanation of the most important components of the mentioned simulation model are given.

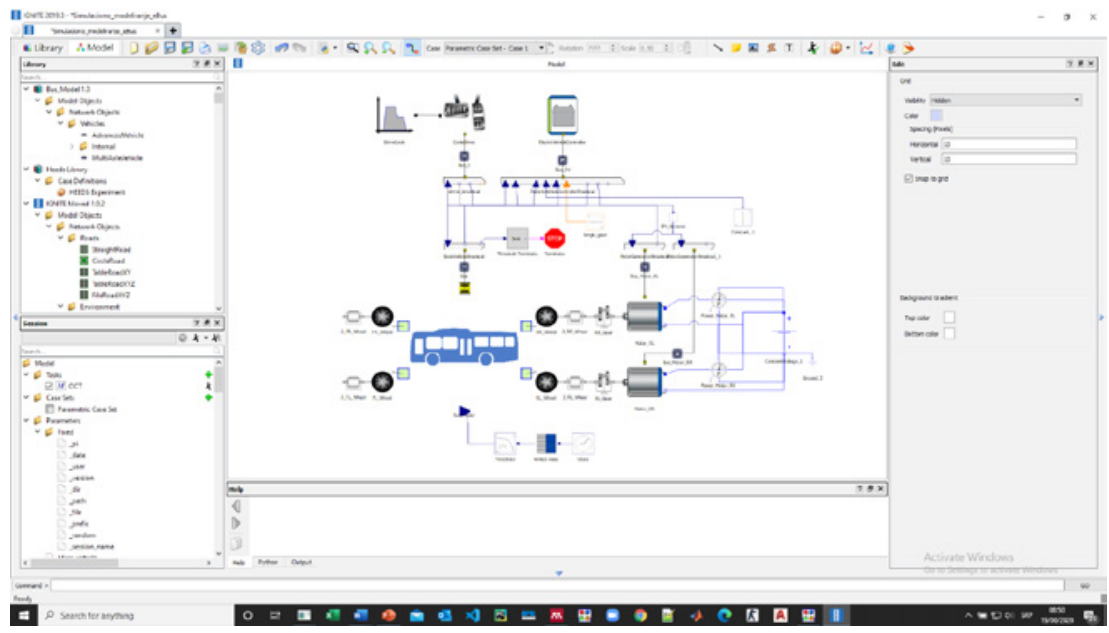

Fig. 2.

Simulation Model of Higer KLQ6125GEV3 e-bus in IGNITE Simulation Software 


\subsection{Vehicle Modeling}

During vehicle modeling, it is necessary to pay attention to importing the real values of the required parameters. Some of the most important parameters that affect the vehicle's technical and operational characteristics in the simulation are vehicle weight, number of axles, distance of center of gravity from the front and rear axles, center of gravity height, maximum braking force, front and rear axle load, suspension system stiffness, front surface vehicles, aerodynamic drag coefficient, etc. Additionally, within the component that depicts the vehicle, it is necessary to define the road profile, i.e. its longitudinal slope as a function of the distance traveled. This can be expressed in tabular form, but since the IGNITE simulation software supports the Modelica modeling language, it is possible to create *.m files that depict the longitudinal slope of the road (bus line) (Figure 3).
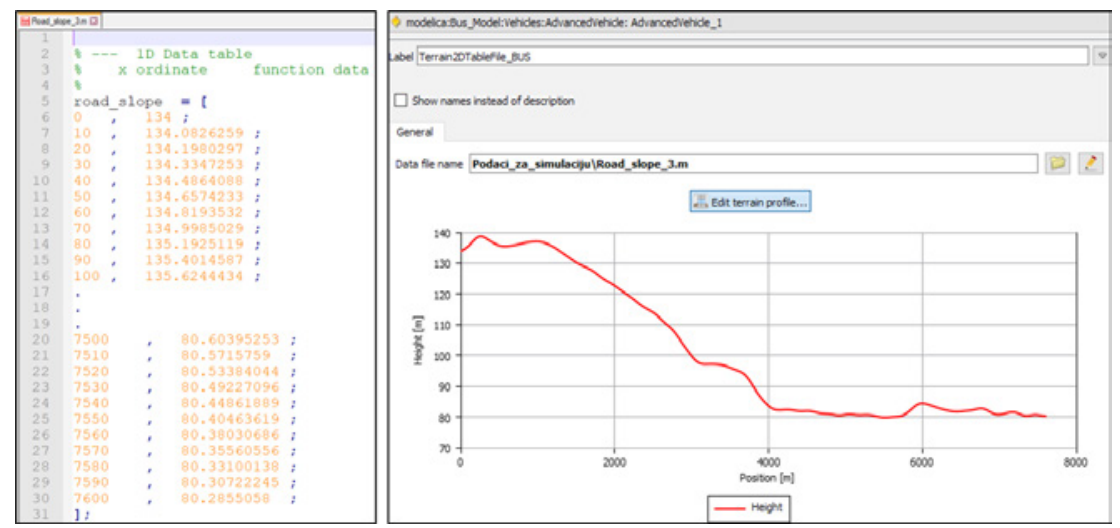

Fig. 3.

Appearance of the File Imported into IGNITE and Appearance of the Longitudinal Slope of the Road in the IGNITE Software

Furthermore, it is necessary to determine the length of the CoG of the empty vehicle from the front axle (x) and the length of the CoG of the passenger compartment from the rear axle (y). In order to do this, it is necessary to determine the sum of the moments around the front or rear axle. Based on the calculation, it was obtained that the length of the CoG of the passenger compartment from the rear axle can be represented by the following equation:

$y=\frac{l_{c a b}}{2}-\left(l_{r o}-l_{b a t}\right)$ while the length of the CoG of the empty vehicle from the front axle is:

$x=\frac{G_{r} * l_{w b}-G_{p} *\left(l_{w b}-y\right)}{G_{o}}$

Where:

- $x$ - length of the CoG of the empty vehicle from the front axle;

- $y$ - length of the CoG of the passenger compartment from the rear axle;

- $G_{0}$ - Curb weight;

- $G_{p}$ - Passenger weight;

- $G$-Gross vehicle weight; 
- $G_{f}$ - Front axle load;

- $G_{r}$ - Rear axle load;

- $l_{c a b}^{r}$ - Passenger space length (cabin);

- $l_{r o}$ - Rear overhang;

- $\quad l_{w b}$ - wheelbase length;
- $l_{\text {bat }}$-supercapacitator length;

- $C o G$ - center of gravity.

Figure 4 shows all parameters (forces and lengths) that are used in the calculation.

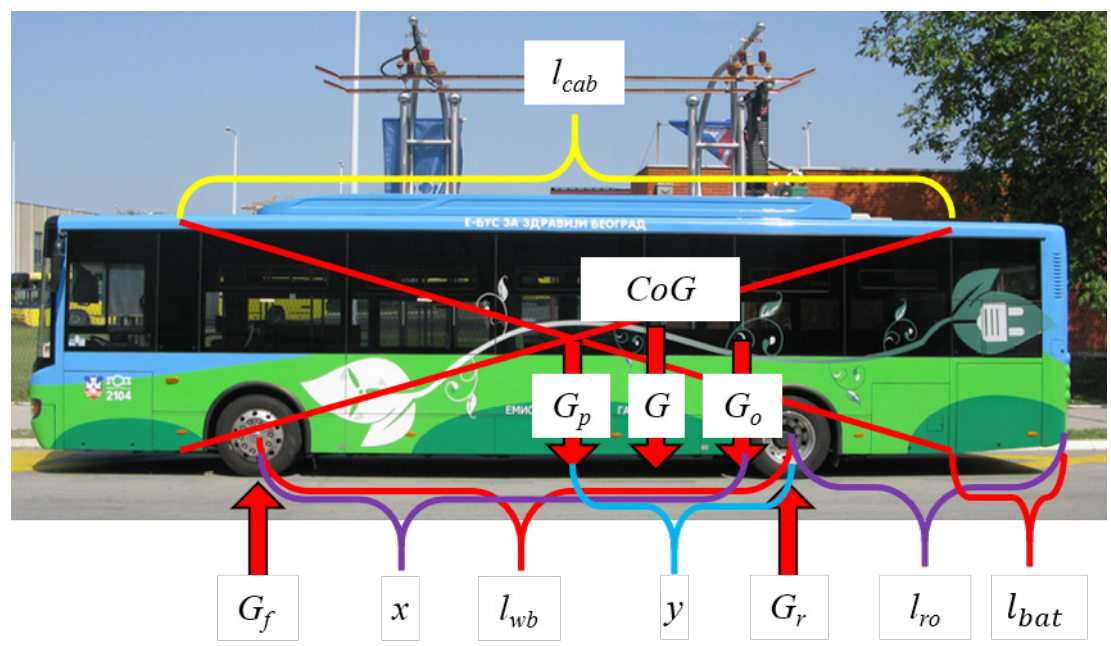

Fig. 4.

Illustrative Presentation of all Listed Parameters (Forces and Lengths) that are applied in the Calculation

\subsection{Electric Motors Modeling}

There are two Siemens' electric motors, model 1PV5135, on the Higer KLQ6125GEV3 bus. Each of these EM has $90 \mathrm{~kW}$ of peak and $60 \mathrm{~kW}$ of nominal power, while the engine torque is $430 \mathrm{Nm}$. In order to successfully perform simulation and determine the current power demand on certain sections of the bus line, it is necessary to properly model the electric motors. In that sense, it is necessary to enter the moments of inertia of the EM, the maximum torques and the maximum power (Figure 5 - left). Furthermore, it is very important to construct efficiency maps of these engines (Figure 5 - right). This map can be constructed by creating *.m files which are then imported into the IGNITE simulation program. 

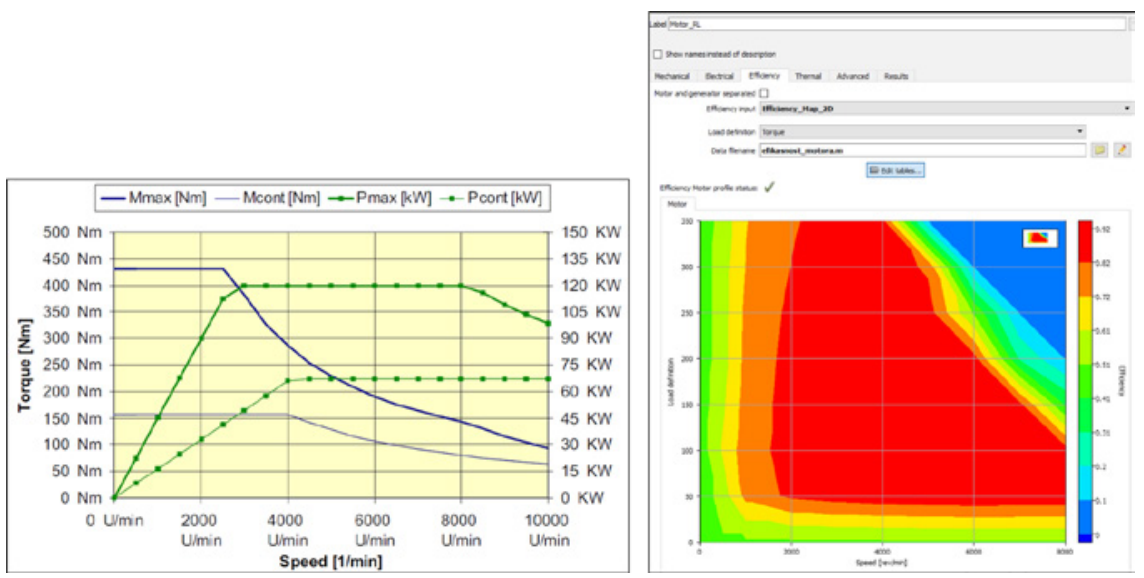

Fig. 5.

Characteristics and Efficiency Map of Siemens 1PV5135 EM

\subsection{Speed Profile Modeling}

One of the most important, and perhaps the most important part when modeling a vehicle in the IGNITE simulation software in order to validate the modeled vehicle, is to create a similar speed profile (drive cycle) as the one obtained in real operating conditions. Data recording in terms of current speed was collected from the CAN bus using HBM QuantumX MX840A device. The determination of the final speed profile was performed in a similar way as the determination of the longitudinal road slope, since there are certain deviations / errors during recording. In that sense, recorded errors had to be corrected or mitigated by applying IGNITE software and the moving average method. Figure 6 shows the initial appearance of the speed profile (orange), as well as the appearance of the speed profile after the IGNITE software and the moving average method have been applied (blue).

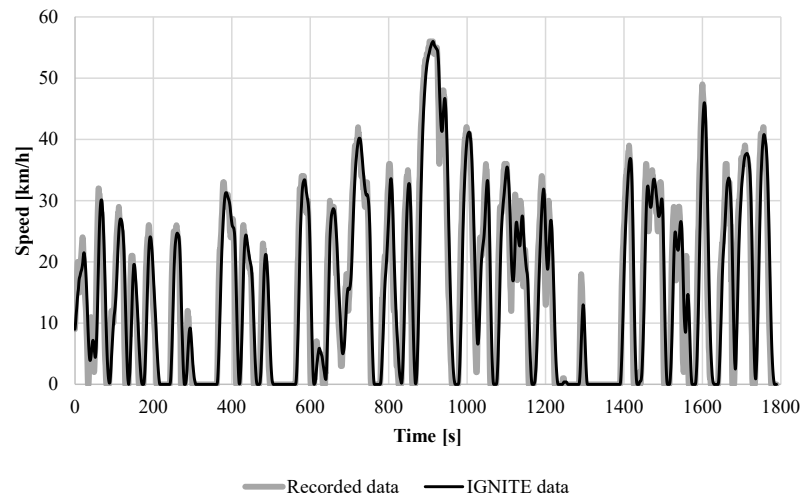

Fig. 6.

Appearance of the Recorded Speed Profile (Grey) and the Appearance of the Speed Profile after the IGNITE Software and the Moving Average Method have been Applied (Black) 


\subsection{Driver's Behavior Modeling "Simulated Driver"}

This section explains in detail the component that represents the driver in the simulation, i.e. the controller that should match the simulated speed with the recorded e-bus speed. The input to this controller is a speed profile that needs to be mapped, while the outputs are deceleration and acceleration demands. Acceleration $\left(\mathrm{a}_{0}\right)$ and deceleration $\left(b_{0}\right)$ requests can be written as follows (Ricardo, 2019):

$$
\begin{aligned}
& a_{0}=C_{f} \cdot\left(C_{a} \cdot a_{t r g}^{+}+C_{r} \cdot \operatorname{sgn}\left(v_{t r g}\right)+C_{v} \cdot v_{t r g}+C_{d} \cdot v_{t r g}^{2}\right)-\left(C_{p} \cdot v_{e r r}+C_{i} \int_{t-\tau}^{t} v_{e r r}(x) d x\right) \\
& b_{0}=B_{f} \cdot\left(C_{a} \cdot a_{t r g}^{+}+C_{r} \cdot \operatorname{sgn}\left(v_{t r g}\right)+C_{v} \cdot v_{t r g}+C_{d} \cdot v_{t r g}^{2}\right)-\left(B_{p} \cdot v_{e r r}+B_{i} \int_{t-\tau}^{t} v_{e r r}(x) d x\right) \\
& a_{t r g}^{+}(t)=\frac{v_{t r g} \cdot(t+d t)-v_{t r g}(t)}{d t}
\end{aligned}
$$

Where:

- $\quad C_{f} ; B_{f}$ - Feedforward coefficient [-];

- $\quad C_{a}$-Acceleration coefficient $\left[\mathrm{s}^{2} / \mathrm{m}\right]$;

- $d t$-Time interval that is observed ahead [s];

- $a_{t r g}^{+}$- Forward coefficient $\left[\mathrm{m} / \mathrm{s}^{2}\right]$;

- $\quad C_{r}$-Rolling resistance coefficient [-];

- $\quad v_{\text {trg }}$ - Desired speed $[\mathrm{m} / \mathrm{s}]$;

- $\quad C_{v}$-Speed resistance coefficient $[\mathrm{s} / \mathrm{m}]$;

- $\quad C_{d}$-Aerodynamic drag coefficient $\left[\mathrm{s}^{2} /\right.$ $\left.\mathrm{m}^{2}\right]$;

- $\quad C_{p} ; B_{p}$ - Proportional coefficient $[\mathrm{s} / \mathrm{m}]$;

- $\quad v_{\text {err }}$ - Deviation from the desired speed $[\mathrm{m} / \mathrm{s}]$;

- $\quad C_{i} ; B_{i}$-Integral coefficient $[1 / \mathrm{m}]$;

- $\int_{t-\tau}^{t} v_{e r r}(x) d x$-Integral of the deviation from the desired speed;

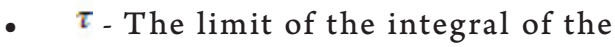
deviation from the desired speed.

The controller works in such a way that it monitors the desired speed profile as a function of time and depending on the current speed and imported speed profile, sends signals from the vehicle to the "simulated driver" whether it is necessary to accelerate or decelerate, and to what extent (acceleration/deceleration request). The controller uses the feedforward model in combination with the feedback model. The feedforward model performs dynamic prediction to determine the required motor load in order to track the required speed profile. Proportional-integral (PI) controllers (one for the acceleration and one for the deceleration), provide detailed feedback control based on the input value from the speed profile (Ricardo, 2019).

Feedback control is generally a sufficient control method, but it is possible to make certain improvements if necessary. The problem with the feedback control lies in the fact that there is no adjustment of the control variable before the control error is different from zero. The reason for this is that this control variable adjusts (changes) when an error occurs, i.e. it acts as a function of the control error. On the other hand, this does not exist with the feedforward control, which can be used as a standalone method, or as a support for the feedback control. In the feedforward control, there is a connection between the input signal, i.e. the input 
value, and the control variable. In that sense feedforward control does no adjustment based on the previous error, but it makes adjustment by observing the upcoming values from the given speed profile and based on the upcoming road characteristics (Ricardo, 2019).

The result of an ideal feedforward control is a zero-control error for all types of signals in observed time, but it is very difficult to achieve zero error since all the values of the variables found in mathematical process models must be known. Regardless that it is very difficult to achieve zero error, by applying the feedforward control, significantly better results (with less error) are obtained than without it. Therefore, feedforward is mainly used with the feedfack control to reduce error due to inaccurate measurements (Ricardo, 2019).

When setting the parameters that define the simulated driver, it is possible to point out several guidelines for their setting. If there are exceedances of the desired speed in the speed profile (higher values than the desired speed are obtained in the acceleration process and lower values than the desired speed are obtained in the deceleration process), it is necessary to increase the proportional coefficient $\left(C_{\mathrm{p}}\right)$. On the other hand, if there are oscillations in the obtained speed profile in the simulation and these oscillations have rapid decreases, it is necessary to reduce the mentioned parameter. If the profile of the obtained speed in the simulation oscillates with a constant amplitude, then it is necessary to reduce the value of the integral coefficient $\left(C_{i}\right)$, while increasing the value of this parameter leads to a constant deviation from the desired speed profile. In order to prevent exceeding the desired speed profile, both in the acceleration process and in the deceleration process, it is necessary to increase the value of the acceleration coefficient $\left(C_{a}\right)$, and the time interval observed in advance $(d t)$ needs to be adjusted according to the acceleration coefficient to obtain the smallest overshoots (Ricardo, 2019).

After defining and modeling all the previously mentioned components (blocks) that make the simulation model, it was necessary to connect them. The verification of the model was carried out by checking whether the blocks used were connected in accordance with the settings of the simulation model, assuming that the components (blocks) of the IGNITE simulation program were previously verified by Ricardo. Since the verification of the model has been successfully performed, it is possible to access the following procedure, which involves the validation of the model, i.e. the procedure for determining whether the simulation model adequately represents the actual system.

\section{Validation of the Simulation Model}

In order to successfully validate the simulation model in terms of generated power, it is necessary to determine an almost identical speed profile. In that sense, the process of defining the values of parameters that affect the speed profile in the simulation is explained below. The synchronization of the recorded speed profile and the speed profile in the simulation was performed through two iterations. In the first iteration, different combinations of feedforward parameters were defined. In that sense, 20 simulations were performed with different values that define the feedforward control in the simulation 
model (Table 2), after which the correlation of the speed profile in the simulation and the recorded speed profile was evaluated. The correlation of the considered speed profiles was performed with the Pearson's correlation coefficient, as well as by determining the absolute and relative error.

Regarding defining coefficients $C_{r}, C_{d}$ and $C_{v}$, it is necessary to calculate their values based on the mass of the vehicle and the maximum possible speed of the vehicle. However, in order to calculate these values accurately, the connection between the engine and the power transmission system must be taken into account so that the torque is the same at all speed intervals. Since the conditions above are very difficult to achieve, it is recommended that these coefficients be equal to zero. In that sense, the values of these coefficients will be set in the simulation model as it is recommended.

Table 2

Values of Parameters $d_{t}$ and $C_{a}$ for each of 20 Conducted Simulations and Values of Correlation Coefficient, Absolute and Relative Error

\begin{tabular}{|l|c|c|c|c|c|}
\hline & $\mathbf{d t}$ & $\mathbf{C}_{\mathbf{a}}$ & $\mathbf{R}^{2}$ & Abs. Error & Rel. Error \\
\hline Simulation 01 & 0.25 & 0 & 0.2628 & 3.0849 & 12.0000 \\
\hline Simulation 02 & 0.25 & 0.25 & 0.2075 & 6.2171 & 12.9300 \\
\hline Simulation 03 & 0.25 & 0.5 & 0.5583 & 2.4648 & 8.6133 \\
\hline Simulation 04 & 0.25 & 0.75 & 0.7011 & 2.4627 & 6.4670 \\
\hline Simulation 05 & $\mathbf{0 . 2 5}$ & $\mathbf{1}$ & $\mathbf{0 . 7 4 4 0}$ & $\mathbf{2 . 6 5 9 7}$ & $\mathbf{6 . 0 3 9 9}$ \\
\hline Simulation 06 & 0.5 & 0 & 0.2626 & 3.0850 & 11.9984 \\
\hline Simulation 07 & 0.5 & 0.25 & 0.2073 & 6.2159 & 12.9218 \\
\hline Simulation 08 & 0.5 & 0.5 & 0.5583 & 2.4628 & 8.6042 \\
\hline Simulation 09 & 0.5 & 0.75 & 0.7003 & 2.4617 & 6.4587 \\
\hline Simulation 10 & 0.5 & 1 & 0.7420 & 2.6573 & 6.0221 \\
\hline Simulation 11 & 0.75 & 0 & 0.2628 & 3.0845 & 11.9992 \\
\hline Simulation 12 & 0.75 & 0.25 & 0.2071 & 6.2140 & 12.9122 \\
\hline Simulation 13 & 0.75 & 0.5 & 0.5578 & 2.4613 & 8.5979 \\
\hline Simulation 14 & 0.75 & 0.75 & 0.6993 & 2.4598 & 6.4557 \\
\hline Simulation 15 & 0.75 & 1 & 0.7398 & 2.6558 & 6.0158 \\
\hline Simulation 16 & 1 & 0 & 0.2628 & 3.0845 & 11.9995 \\
\hline Simulation 17 & 1 & 0.25 & 0.2069 & 6.2126 & 12.9024 \\
\hline Simulation 18 & 1 & 0.5 & 0.5576 & 2.4600 & 8.5955 \\
\hline Simulation 19 & 1 & 0.75 & 0.6981 & 2.4578 & 6.4578 \\
\hline Simulation 20 & 1 & 1 & 0.7374 & 2.6538 & 6.0114 \\
\hline
\end{tabular}

Since the setting of the feedforward model only is not enough to achieve a sufficiently good correlation between recorded and speed profile in the simulation it is necessary to find the values of the parameters that control acceleration and deceleration. The values of the parameters adopted for defining the feedforward model in the simulation are the values that gave the best results of correlation coefficient in first 20 simulations $(d t=0.25$ and $C_{a}=1$ ).

Furthermore, within the second iteration, different values of the integral coefficient $\left(C_{i}\right)$, proportional coefficient $\left(C_{p}\right)$ and feedforward coefficient $\left(C_{f}\right)$ were defined for each of the 
75 performed simulations (Table 3). After defining the mentioned parameters, as in the previous simulations, the evaluation of the correlation of the obtained speed profile in the simulation with the actual speed profile was performed. In this case as well, the evaluation of the correlation of the observed speed profiles was performed with the Pearson's correlation coefficient, as well as with the calculation of the absolute and relative error.

Table 3

Values of Parameters $C_{i}, C_{p}$ and $C_{f}$ for each of 75 Conducted Simulations

\begin{tabular}{|c|c|c|c|c|c|c|c|c|c|c|}
\hline Simulation no. & S 1 & S 2 & S 3 & S 4 & S 5 & S 6 & S 7 & S 8 & S9 & S 10 \\
\hline $\mathrm{C}_{i} / \mathrm{B}_{i}$ & 0 & 0.25 & 0.5 & 0.75 & 1 & 0 & 0.25 & 0.5 & 0.75 & 1 \\
\hline $\mathrm{C}_{p} / \mathrm{B}_{p}$ & 0 & 0 & 0 & 0 & 0 & 0.25 & 0.25 & 0.25 & 0.25 & 0.25 \\
\hline $\mathbf{C}_{f} / \mathbf{B}_{f}$ & 0 & 0 & 0 & 0 & 0 & 0 & 0 & 0 & 0 & 0 \\
\hline Simulation no. & S 11 & S 12 & S 13 & S 14 & S 15 & S 16 & S 17 & S 18 & S 19 & S 20 \\
\hline $\mathbf{C}_{i} / \mathbf{B}_{i}$ & 0 & 0.25 & 0.5 & 0.75 & 1 & 0 & 0.25 & 0.5 & 0.75 & 1 \\
\hline $\mathbf{C}_{p} / \mathbf{B}_{p}$ & 0.5 & 0.5 & 0.5 & 0.5 & 0.5 & 0.75 & 0.75 & 0.75 & 0.75 & 0.75 \\
\hline $\mathbf{C}_{f} / \mathbf{B}_{f}$ & 0 & 0 & 0 & 0 & 0 & 0 & 0 & 0 & 0 & 0 \\
\hline Simulation no. & S 21 & S 22 & S 23 & S 24 & S 25 & S26 & S 27 & S 28 & S 29 & S 30 \\
\hline $\mathrm{C}_{i} / \mathrm{B}_{i}$ & 0 & 0.25 & 0.5 & 0.75 & 1 & 0 & 0.25 & 0.5 & 0.75 & 1 \\
\hline $\mathbf{C}_{p} / \mathbf{B}_{p}$ & 1 & 1 & 1 & 1 & 1 & 0 & 0 & 0 & 0 & 0 \\
\hline $\mathbf{C}_{f} / \mathbf{B}_{f}$ & 0 & 0 & 0 & 0 & 0 & 0.5 & 0.5 & 0.5 & 0.5 & 0.5 \\
\hline Simulation no. & S 31 & S 32 & S 33 & S 34 & S 35 & S 36 & S 37 & S 38 & S 39 & S 40 \\
\hline $\mathbf{C}_{i} / \mathbf{B}_{i}$ & 0 & 0.25 & 0.5 & 0.75 & 1 & 0 & 0.25 & 0.5 & 0.75 & 1 \\
\hline $\mathrm{C}_{p} / \mathrm{B}_{p}$ & 0.25 & 0.25 & 0.25 & 0.25 & 0.25 & 0.5 & 0.5 & 0.5 & 0.5 & 0.5 \\
\hline $\mathbf{C}_{f} / \mathbf{B}_{f}$ & 0.5 & 0.5 & 0.5 & 0.5 & 0.5 & 0.5 & 0.5 & 0.5 & 0.5 & 0.5 \\
\hline Simulation no. & S 41 & S 42 & S 43 & S 44 & S 45 & S 46 & S 47 & S 48 & S 49 & S 50 \\
\hline $\mathrm{C}_{i} / \mathrm{B}_{i}$ & 0 & 0.25 & 0.5 & 0.75 & 1 & 0 & 0.25 & 0.5 & 0.75 & 1 \\
\hline $\mathrm{C}_{p} / \mathrm{B}_{p}$ & 0.75 & 0.75 & 0.75 & 0.75 & 0.75 & 1 & 1 & 1 & 1 & 1 \\
\hline $\mathbf{C}_{f} / \mathbf{B}_{f}$ & 0.5 & 0.5 & 0.5 & 0.5 & 0.5 & 0.5 & 0.5 & 0.5 & 0.5 & 0.5 \\
\hline Simulation no. & S 51 & S 52 & S53 & S54 & S 55 & S56 & $\mathbf{S 5 7}$ & \begin{tabular}{|l|} 
S 58 \\
\end{tabular} & S 59 & 560 \\
\hline $\mathrm{C}_{i} / \mathrm{B}_{i}$ & 0 & 0.25 & 0.5 & 0.75 & 1 & 0 & 0.25 & 0.5 & 0.75 & 1 \\
\hline $\mathbf{C}_{p} / \mathbf{B}_{p}$ & 0 & 0 & 0 & 0 & 0 & 0.25 & 0.25 & 0.25 & 0.25 & 0.25 \\
\hline $\mathbf{C}_{f} / \mathbf{B}_{f}$ & 1 & 1 & 1 & 1 & 1 & 1 & 1 & 1 & 1 & 1 \\
\hline Simulation no. & S 61 & S 62 & 563 & S 64 & S65 & S66 & S 67 & S 68 & S 69 & S 70 \\
\hline $\mathbf{C}_{i} / \mathbf{B}_{i}$ & 0 & 0.25 & 0.5 & 0.75 & 1 & 0 & 0.25 & 0.5 & 0.75 & 1 \\
\hline $\mathbf{C}_{p} / \mathbf{B}_{p}$ & 0.5 & 0.5 & 0.5 & 0.5 & 0.5 & 0.75 & 0.75 & 0.75 & 0.75 & 0.75 \\
\hline $\mathbf{C}_{f} / \mathbf{B}_{f}$ & 1 & 1 & 1 & 1 & 1 & 1 & 1 & 1 & 1 & 1 \\
\hline Simulation no. & S 71 & S 72 & S 73 & S 74 & S 75 & & & & & \\
\hline $\mathrm{C}_{i} / \mathrm{B}_{i}$ & 0 & 0.25 & 0.5 & 0.75 & 1 & & & & & \\
\hline $\mathrm{C}_{p} / \mathrm{B}_{p}$ & 1 & 1 & 1 & 1 & 1 & & & & & \\
\hline $\mathbf{C}_{f} / \mathbf{B}_{f}$ & 1 & 1 & 1 & 1 & 1 & & & & & \\
\hline
\end{tabular}

Based on the obtained results through 75 simulations (Table 4), it can be noticed that the best match was obtained in the simulation 75 where the values of the parameters $C_{i}=C_{p}$
$=C_{f}=B_{i}=B_{p}=B_{f}=1$. In this case the speed profile and speed profile in the simulation and recorded speed profile have the smallest deviation, i.e. the highest correlation 
coefficient $\left(R^{2}=0.9997\right)$. Additionally, the obtained speed profile in this simulation has the lowest average value of the absolute error, and the relative error is only $4 \%$.

Table 4

Values of Correlation Coefficient, Absolute Error and Relative Error for each of 75 Conducted Simulations

\begin{tabular}{|c|c|c|c|c|c|c|c|c|c|c|}
\hline Simulation no. & S 1 & S 2 & S 3 & S 4 & S 5 & S 6 & S 7 & S 8 & S 9 & S 10 \\
\hline $\mathbf{R}^{2}$ & 0.2761 & 0.9639 & 0.9811 & 0.9903 & 0.9867 & 0.9314 & 0,9938 & 0,9979 & 0,9987 & 0,9991 \\
\hline Abs. error & 4.0562 & 0.4737 & 0.3162 & 0.2327 & 0.2655 & 0.7313 & 0,2009 & 0,1098 & 0,0799 & 0,0627 \\
\hline Rel. error & 0.8155 & 0.2446 & 0.1955 & 0.1707 & 0.1707 & 1.4546 & 0,1339 & 0,0967 & 0,0815 & 0,0714 \\
\hline Simulation no. & S 11 & S 12 & S 13 & $S 14$ & S 15 & $S 16$ & S 17 & S 18 & S 19 & S 20 \\
\hline $\mathbf{R}^{2}$ & 0.9777 & 0.9963 & 0.9985 & 0.9991 & 0.9994 & 0.9892 & 0,9973 & 0,9988 & 0,9903 & 0,9903 \\
\hline Abs. error & 0.4100 & 0.1603 & 0.0942 & 0.0673 & 0.0529 & 0.2840 & 0,1355 & 0,0856 & 0,2326 & 0,2654 \\
\hline Rel. error & 0.5559 & 0.1152 & 0.0887 & 0.0721 & 0.0640 & 0.3211 & 0,1025 & 0,0830 & 0,1707 & 0,1706 \\
\hline Simulation no. & S 21 & S 22 & S 23 & S 24 & S 25 & S 26 & S 27 & S 28 & S 29 & S 30 \\
\hline $\mathbf{R}^{2}$ & 0.9936 & 0.9980 & 0.9990 & 0.9993 & 0.9995 & 0.5665 & 0,9822 & 0,9913 & 0,9917 & 0,9904 \\
\hline Abs. error & 0.2170 & 0.1179 & 0.0788 & 0.0588 & 0.0471 & 1.9303 & 0,3169 & 0,2138 & 0,1899 & 0,2010 \\
\hline Rel. error & 0.2226 & 0.0922 & 0.0780 & 0.0670 & 0.0589 & 2.9833 & 0,2268 & 0,1933 & 0,1467 & 0,1827 \\
\hline Simulation no. & S 31 & S 32 & S 33 & S 34 & S 35 & $S 36$ & S 37 & S 38 & S 39 & S 40 \\
\hline $\mathbf{R}^{2}$ & 0.9819 & 0.9979 & 0.9991 & 0.9993 & 0.9994 & 0.9939 & 0,9987 & 0,9993 & 0,9995 & 0,9996 \\
\hline Abs. error & 0.3629 & 0.1110 & 0.0637 & 0.0466 & 0.0381 & 0.2071 & 0,0862 & 0,0530 & 0,0391 & 0,0316 \\
\hline Rel. error & 0.7169 & 0.0931 & 0.0721 & 0.0657 & 0.0722 & 0.3079 & 0,0785 & 0,0619 & 0,0530 & 0,0502 \\
\hline Simulation no. & S 41 & S 42 & S 43 & S 44 & S 45 & S 46 & S 47 & S 48 & S 49 & S 50 \\
\hline $\mathbf{R}^{2}$ & 0.9969 & 0.9991 & 0.9995 & 0.9996 & 0.9996 & 0.9981 & 0,9993 & 0,9995 & 0,9996 & 0,9997 \\
\hline Abs. error & 0.1467 & 0.0721 & 0.0475 & 0.0356 & 0.0289 & 0.1136 & 0,0624 & 0,0434 & 0,0333 & 0,0273 \\
\hline Rel. error & 0.1911 & 0.0694 & 0.0563 & 0.0478 & 0.0420 & 0.1380 & 0,0621 & 0,0524 & 0,0455 & 0,0402 \\
\hline Simulation no. & S 51 & S 52 & $S 53$ & S 54 & S 55 & S 56 & S 57 & S 58 & S 59 & S 60 \\
\hline $\mathbf{R}^{2}$ & 0.5961 & 0.9922 & 0.9924 & 0.9927 & 0.9927 & 0.9820 & 0,9984 & 0,9993 & 0,9995 & 0,9995 \\
\hline Abs. error & 2.3787 & 0.2369 & 0.2008 & 0.1888 & 0.1923 & 0.3773 & 0,0924 & 0,0503 & 0,0365 & 0,0308 \\
\hline Rel. error & 3.7653 & 0.3741 & 0.2264 & 0.2210 & 0.2451 & 0.3443 & 0,1116 & 0,0863 & 0,0743 & 0,0638 \\
\hline Simulation no. & S 61 & S 62 & S 63 & S 64 & S 65 & S 66 & S 67 & S 68 & S 69 & S 70 \\
\hline $\mathbf{R}^{2}$ & 0.9945 & 0.9990 & 0.9995 & 0.9996 & 0.9997 & 0.9974 & 0,9993 & 0,9996 & 0,9997 & 0,9997 \\
\hline Abs. error & 0.2046 & 0.0735 & 0.0430 & 0.0316 & 0.0258 & 0.1412 & 0,0630 & 0,0394 & 0,0293 & 0,0239 \\
\hline Rel. error & 0.2126 & 0.0883 & 0.0672 & 0.0316 & 0.0518 & 0.1573 & 0,0729 & 0,0580 & 0,0504 & 0,0453 \\
\hline Simulation no. & S 71 & S 72 & S 73 & S 74 & S 75 & & & & & \\
\hline $\mathbf{R}^{2}$ & 0.9984 & 0.9994 & 0.9996 & 0.9997 & 0.9997 & & & & & \\
\hline Abs. error & 0.1082 & 0.0558 & 0.0368 & 0.0278 & 0.0228 & & & & & \\
\hline Rel. error & 0.1258 & 0.0626 & 0.0517 & 0.0453 & 0.0410 & & & & & \\
\hline
\end{tabular}

The correlation of the obtained speed profile in the simulation and the actual speed profile is given in Figure 7. From this figure it can be seen that the speed profile in the simulation is almost completely identical to the desired speed profile, with very small, almost insignificant deviations. In that sense, based on this speed profile, the simulation model of electric bus Higer KLQ6125GEV3 was validated in terms of the generated power on the EM. 


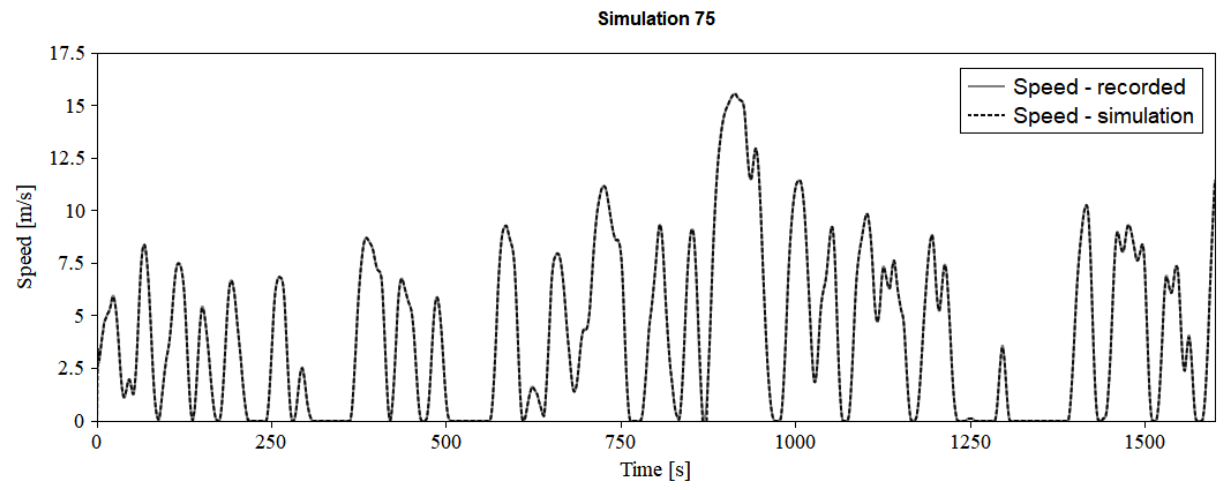

Fig. 7.

Speed Profiles with the Best Correlation Coefficient

Furthermore, the correlation between the generated power recorded in real operating conditions and the generated power obtained in the simulation software is shown in Figure 8. Considering that the power on the chart is expressed in watts (W), and that the deviations are very small (the degree of correlation is $87.5 \%$ ), it can be said that the Higer KLQ6125GEV3 bus model modeled in the IGNITE simulation software is credible to the real bus model. It can be noticed that perhaps the largest deviations are the extreme values of the recorded power, but this can be attributed to both measurement errors and a very dynamic change of power in time. Additionally, it is necessary to emphasize once again that the power on the chart is expressed in watts, while in real operating conditions the power is observed in kilowatts $(\mathrm{kW})$ and is considered as a very unstable value, so in this sense these deviations are very small, almost negligible. Regarding this, it can be said that the simulation model of e-bus Higer KLQ6125GEV3 is validated.

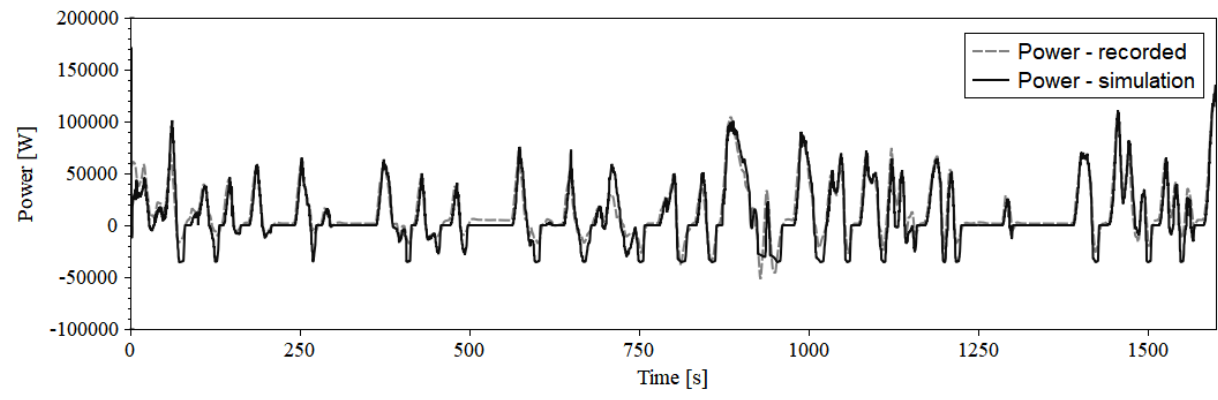

Fig. 8.

Correlation of Generated Power in Real Operating Conditions and Generated Power obtained in the Simulation Model 


\section{Conclusions}

In the paper the simulation model of an electric bus Higer KLQ6125GEV3 was developed in the simulation software IGNITE. The technical and operational characteristics of the observed bus are defined, as well as the characteristics of the bus line on which this vehicle operates. The main contribution of this paper is validation of the modeled bus in terms of generated power as a function of time and distance traveled, which was achieved by adjusting the parameters of the simulation model components through almost 100 simulations. As a final result, a verified and validated simulation model of the mentioned bus was realized, where the correlation coefficient between the speed profile obtained by simulation and the recorded speed profile in real-world operating conditions was $99.97 \%$ and relative error was $4 \%$, while the correlation coefficient between generated power in simulation and generated power in real operating conditions was $87.5 \%$.

As for the directions of future research, it is possible to conduct a more detailed analysis of vehicle components and determine the values of the parameters that define them, in order to obtain smaller deviations in terms of generated power. After that, the model itself can be applied on some other lines of public transport to determine how much power and energy is needed to implement this vehicle on them. Additionally, it is possible to analyze the energy consumption on the observed bus line depending on the different driver's behavior. In this sense, it is possible to determine the potential savings that can be achieved when applying certain principles and techniques of eco-driving, such as a rule of gentle acceleration and deceleration.

\section{Acknowledgements}

The authors would like to express their gratitude to Ricardo for the opportunity to work in the IGNITE simulation software, without which this research could not have been realized. This paper was partially supported by the Ministry of Education, Science and Technological Development of the Republic of Serbia through projects TR 36010 and TR 44004.

\section{References}

Emberger, G. 2017. Low Carbon Transport Strategy in Europe: A Critical Review, International Journal of Sustainable Transportation 11(1): 31-35.

Eurostat. 2019. Freight transport statistics - modal split: tables and figures. Available from Internet: <https://ec.europa.eu/eurostat/statistics-explained/ pdfscache/1142.pdf $>$.

Fu, P.; Pudjianto, D.; Zhang, X.; Strbac, G. 2019. Evaluating Strategies for Decarbonising the Transport Sector in Great Britain. In Proceedings of the 2019 IEEE Milan PowerTech, 1-6.

Huang, F.; Zhou, D.; Wang, Q., Hang, Ye. 2019. Decomposition and Attribution Analysis of the Transport Sector's Carbon Dioxide Intensity Change in China, Transportation Research Part A: Policy and Practice 119: 343-58. https://doi.org/10.1016/j.tra.2018.12.001.

Kumar, A.; Vijay, S.; Kumar, R., Patil, R.S.; Dikshit. A.K.; Dhingra, S. L. 2018. Prediction and Analysis of Pollution and Congestion Level for Present and Future Scenario on an Urban Road Network-India, International Journal for Traffic and Transport Engineering 8(2): 213-22.

Mišanović, S.; Živanović, Z.; Tica, S. 2015. Energy Efficiency of Different Bus Subsystems in Belgrade Public Transport, Thermal Science 19(6): 2233-44. 
Mišanović, S.; Milković, Ž.; Savković, D.; Krstić, P.; Stević, S. 2018. Speciticity of Exploitation and Maintenance of Electric Buses in CTC Belgrade. In Proceedings of the Maintenance Forum 2018, 119-27.

RDW. 2014. EC Type-Approval Certificate (for a vehicle) Higer E-Bus KLQ6125GEV3. Vehicle Technology Division, Netherlands.

Ricardo. 2019. Ricardo Software. IGNITE User Manual.

Solaymani, S. 2019. $\mathrm{CO}_{2}$ Emissions Patterns in 7 Top Carbon Emitter Economies: The Case of Transport Sector, Energy 168: 989-1001.

Torok, A. 2015. Monte-Carlo Simulation of Road Transport Emission, International Journal for Traffic and Transport Engineering 5(3): 278-85.

UITP. 2016. ZeUS EBus Report: An Overview of Electric Buses in Europe. Available from Internet: <http://zeeus.eu/uploads/publications/documents/ zeeus-ebus-report-internet.pdf $>$.
Velazquez, L.; Munguia, N.E.; Will, M.; Zavala, A.G.; Verdugo, S.P.; Delakowitz, B.; Giannetti, B. 2015. Sustainable Transportation Strategies for Decoupling Road Vehicle Transport and Carbon Dioxide Emissions, Management of Environmental Quality: An International Journal 26(3): 373-388.

Walmsley, M.R.W.; Walmsley, T.G.; Atkins, M.J.; Kamp, P.J.J.; Neale, J.R.; Chand, A. 2015. Carbon Emissions

Pinch Analysis for Emissions Reductions in the New Zealand Transport Sector through to 2050, Energy 92: 569-76.

Xylia, M.; Silveira, S. 2017. On the Road to Fossil-Free Public Transport: The Case of Swedish Bus Fleets, Energy Policy 100: 397-412. 